\title{
TIAM1 inhibits lung fibroblast differentiation in pulmonary fibrosis
}

\author{
ZHICHENG HUANG $^{1 *}$, SHUANGYAN SUN ${ }^{1 *}$, CHANGLIANG YANG $^{2}$, JUN ZHENG $^{1}$, \\ YINGJI NAN ${ }^{1}$, RUIKUN ZHAO $^{1}$, ZHIGUO LANG $^{1}$, HANG $^{3}{ }^{3}$ and LIXIA MA ${ }^{2}$ \\ Departments of ${ }^{1}$ Radiology and ${ }^{2}$ Thoracic Oncology; ${ }^{3}$ The First Division of \\ Chest Medicine, Jilin Province Cancer Hospital, Changchun, Jilin 130012, P.R. China
}

Received July 19, 2016; Accepted June 16, 2017

DOI: $10.3892 / \mathrm{etm} .2017 .5024$

\begin{abstract}
The differentiation of fibroblasts to myofibroblasts is critical for the development of idiopathic pulmonary fibrosis (IPF). T-cell lymphoma invasion and metastasis 1 (TIAM1) is known to be associated with amyotrophic lateral sclerosis 1 and colorectal cancer; however, its role in IPF is unclear. The aim of the present study was to investigate the expression and roles of TIAM1 in lung fibroblasts during pulmonary fibrosis. It was demonstrated that TIAM1 expression was significantly increased in fibrotic lung tissue and lung fibroblasts from bleomycin (BLM)-treated mice compared with control mice $(\mathrm{P}<0.05)$. TIAM1 expression and differentiation were significantly upregulated in human lung fibroblasts challenged with transforming growth factor- $\beta$ (TGF- $\beta$ ) compared with unchallenged cells $(\mathrm{P}<0.05)$. Furthermore, inhibition of the nuclear factor (NF)- $\mathrm{kB}$ signaling pathway significantly attenuated TGF- $\beta$-induced TIAM1 expression and decreased fibroblast differentiation in human lung fibroblasts $(\mathrm{P}<0.05)$. Similarly, overexpression of TIAM1 significantly inhibited TGF- $\beta$-induced fibroblast differentiation, as indicated by decreased expression of fibronectin and $\alpha$-smooth muscle actin
\end{abstract}

Correspondence to: Dr Lixia Ma, Department of Thoracic Oncology, Jilin Province Cancer Hospital, 1018 Lake Road, Changchun, Jilin 130012, P.R. China

E-mail: lixiama1234567@163.com

Dr Hang Li, The First Division of Chest Medicine, Jilin Province Cancer Hospital, 1018 Lake Road, Changchun, Jilin 130012, P.R. China

E-mail: dentonlee@163.com

*Contributed equally

Abbreviations: TIAM1, T-cell lymphoma invasion and metastasis 1; IPF, idiopathic pulmonary fibrosis; FN, fibronectin; ECM, extracellular matrix; $\alpha$-SMA, $\alpha$-smooth muscle actin; DMEM, Dulbecco's modified Eagle's medium; TGF- $\beta$, transforming growth factor- $\beta$; BLM, bleomycin

Key words: T-cell lymphoma invasion and metastasis 1, idiopathic pulmonary fibrosis, transforming growth factor- $\beta$, fibroblast
(SMA; $\mathrm{P}<0.05)$. The results of the present study also demonstrated that TIAM1 knockdown increased TGF- $\beta$-induced fibroblast differentiation $(\mathrm{P}<0.05)$. These findings suggest that TIAM1 expression is associated with lung fibroblast differentiation in pulmonary fibrosis via an NF- $\mathrm{kB}$-dependent pathway, and that TIAM1 inhibits lung fibroblast differentiation in pulmonary fibrosis.

\section{Introduction}

Human T-lymphoma invasion and metastasis-inducing protein 1 (TIAM1) is 1,591 amino acids long with a molecular weight of $177 \mathrm{kD}(1)$, and is detected at the boundary between cells with actin-rich protrusions (2). TIAM1 has previously been demonstrated to modulate the activity of Rho-like proteins and connect extracellular signals to cytoskeletal activities through guanosine diphosphate-guanosine triphosphate exchange activity (3). TIAM1 serves a critical role in the regulation of cell adhesion, invasion and migration, which have been directly implicated in the promotion of cancer progression and metastasis (4). A previous clinical investigation in patients with lung cancer has indicated that TIAM1 protein expression in lung tumor tissue is significantly higher compared with that in normal lung tissue, and the expression of TIAM1 is correlated with patient age, tumor size, tumor type and tumor differentiation (5). In human lung cancer cells, decreased expression of TIAM1 due to ubiquitination-mediated degradation or short interfering RNA (siRNA)-mediated knockdown has been reported to induce the disassembly of cell junctions and cancer cell invasion (6). Furthermore, increased expression of TIAM1 induces human lung cancer cell migration (4). In an orthotropic nude mouse model of colon cancer, TIAM1 expression was reported to be upregulated in colon carcinoma growths at metastatic sites, suggesting that overexpression of TIAM1 contributes to the metastatic phenotype of colon cancer cells (7). It has previously been implicated that TIAM1 is a crucial component of the Par complex in the regulation of neuronal and epithelial polarity (8). In normal lung tissue, mechanical stretch decreases the migration of alveolar epithelial cells via the induction of TIAM1 translocation from the membrane to the cytosol, suggesting that TIAM1 is involved in the repair mechanisms of alveolar epithelial type 2 cells subjected to mechanical strain (9). However, the role of 
TIAM1 in lung fibroblasts during pulmonary fibrosis has not been widely studied.

Idiopathic pulmonary fibrosis (IPF) affects more than three million people worldwide (494.5 cases/100,000 people), and half of these patients will succumb within 2-5 years of the initial diagnosis due to limited effective treatments (10). Myofibroblasts are thought to be the key effector cells in IPF and have several potential sources, including epithelial to mesenchymal cell transition, resident fibroblast differentiation and recruitment of circulating fibrocytes (11). Lung fibroblast proliferation, differentiation and accumulation in fibrotic foci serve major roles in pulmonary fibrosis, stimulating the deposition of extracellular matrix (ECM) proteins, including fibronectin (FN) and collagen, in alveoli and resulting in lung dysfunction (12). A previous study indicated that various cytokines and lipid ligands regulate lung fibroblast proliferation, differentiation and invasion (13). Transforming growth factor- $\beta$ (TGF- $\beta$ ) is one of the key factors that induce fibroblast differentiation and lung fibrosis (14). Adenovirus-induced TGF- $\beta 1$ overexpression in a mouse model of lung injury has been reported to induce pulmonary fibrosis (15). Typically, TGF- $\beta 1$ signaling proceeds via the activation of type I and type II serine/threonine kinase receptors, which phosphorylates and translocates mothers against decapentaplegic (Smad)2 and Smad3 to the nucleus to stimulate gene expression in lung fibroblasts (16). TGF- $\beta 1$ also activates nuclear factor (NF)- $\kappa \mathrm{B}$-dependent pathways to induce gene expression in lung fibroblasts during pulmonary fibrosis (17).

In the present study, a bleomycin (BLM)-induced mouse model of pulmonary fibrosis was used to investigate whether the expression of TIAM1 was increased in lung fibroblasts from lung fibrotic foci. The results indicated that in vitro the expression of TIAM1 in TGF- $\beta 1$-challenged fibroblasts depends in part on the NF- $\mathrm{B}$-mediated pathway, and overexpression of TIAM1 attenuates TGF- $\beta 1$-induced lung fibroblast differentiation. In summary, the results of the present study indicate that TIAM1 is critical for fibroblast differentiation and pulmonary fibrosis.

\section{Materials and methods}

Antibodies and reagents. Mouse anti- $\alpha$-smooth muscle actin ( $\alpha$-SMA; cat. no. A5228), anti- $\beta$-actin antibodies (cat. no. A5441), Bay 11-7082 (NF-кB inhibitor; cat. no. B5556) and protease inhibitor cocktail tablets (EDTA-free Complete) were purchased from Sigma-Aldrich (Merck KGaA, Darmstadt, Germany). Recombinant human TGF- $\beta 1$ was obtained from PeproTech, Inc. (Rocky Hill, NJ, USA). Cell lysis buffer and mouse anti-fibroblast specific protein 1 (FSP1; cat. no. 13018) antibody were purchased from Cell Signaling Technology, Inc. (Danvers, MA, USA). Rabbit anti-FN (cat. no. sc-69681), anti-TIAM1 (cat. no. sc-872), anti-GAPDH (cat. no. sc-47724), control mouse IgG (cat. no. sc-2025) and control rabbit IgG (cat. no. sc-2051) antibodies were all purchased from Santa Cruz Biotechnology, Inc. (Dallas, TX, USA). The open reading frame clone of human TIAM1 (cat. no. 70631) and the control plasmid (pCDNA3.1; cat. no. 63560) were purchased from Addgene, Inc. (Cambridge, MA, USA). Horseradish peroxidase-conjugated anti-mouse IgG (cat. no. 170-6515) and anti-rabbit IgG (cat. no. 170-6516) antibodies were obtained from Bio-Rad Laboratories, Inc. (Hercules, CA, USA).

Mouse model of pulmonary fibrosis. A total of 20 wild-type (C57BL/6J) mice (male, 8-10 weeks, 20 g) were purchased from Vital River Laboratory Animal Technology (Beijing, China). Mice were provided with free access to food and water, and housed under a $12 \mathrm{~h}$ light/dark cycle at $18-23^{\circ} \mathrm{C}$ and $40-60 \%$ humidity. Mice were randomly divided into two groups; BLM $(n=15)$ and negative control $(n=5)$. Mice were anesthetized (IP, $87.5 \mathrm{mg} / \mathrm{kg}$ of ketamine and $12.5 \mathrm{mg} / \mathrm{kg}$ of xylazine; Sigma-Aldrich; Merck KGaA, Darmstadt, Germany) and then an intratracheal injection of BLM (2 U/kg; Sigma-Aldrich; Merck KGaA) in saline (50 $\mu \mathrm{l})$ or saline alone was administered (18). At 21 days following the BLM challenge, mice were sacrificed. Lungs were harvested and the lobes were fixed at $25^{\circ} \mathrm{C}$ in $10 \%$ formalin for $24 \mathrm{~h}$ and $70 \%$ EtOH for $48 \mathrm{~h}$, embedded in paraffin, cut into $5-\mu \mathrm{m}$ sections and subjected to hematoxylin and eosin staining $\left(25^{\circ} \mathrm{C} 4 \mathrm{~min}\right.$ hematoxylin and $2 \mathrm{~min}$ eosin) and trichrome staining $\left(25^{\circ} \mathrm{C}\right.$, trichrome, $10 \mathrm{~min})$. All animal studies were approved by the Animal Care Use Committee of Jilin Province Cancer Hospital (Changchun, China).

Immunofluorescence microscopy. Immunofluorescence microscopy was used to assess the expression of ECM-related protein and TIAM1 as previously described (19). Briefly, paraffin-embedded mouse lung tissue sections were dewaxed, rehydrated and subjected to antigen retrieval. Antigen retrieval of tissue slides was performed according to the EDTA buffer antigen retrieval protocol, in which tissue slides were immersed in EDTA buffer containing $1 \mathrm{mM}$ EDTA and $0.05 \%$ Tween-20 (pH 8.0) at $95-100^{\circ} \mathrm{C}$ for $30 \mathrm{~min}$. Sections were subsequently blocked with TBST blocking buffer $[2 \%$ bovine serum albumin (BSA) and $1 \%$ fetal bovine serum (FBS); Santa Cruz Biotechnology, Inc.] for $30 \mathrm{~min}$ at room temperature. Lung tissues were then incubated with primary antibodies (1:200, rabbit anti-TIAM1 (cat. no. sc-872; Santa Cruz Biotechnology, Inc.), mouse anti- $\alpha$-SMA (cat. no. A5228; Sigma-Aldrich; Merck KGaA), control IgG from mouse (cat. no. sc-2025) and rabbit (cat. no. sc-2051) (both from Santa Cruz Biotechnology, Inc.) for $1 \mathrm{~h}$, followed by three 15-min washes with TBST. After this, tissues were stained with Alexa Fluor secondary antibodies (1:200, cat. no. R37117 and A-21202; Thermo Fisher Scientific, Inc., Waltham, MA, USA) at $25^{\circ} \mathrm{C}$ for $1 \mathrm{~h}$, followed by washing with TBST for $15 \mathrm{~min}$. Slides were incubated with mounting media (containing DAPI) at $25^{\circ} \mathrm{C}$ for $10 \mathrm{~min}$, and examined under a Nikon Eclipse TE2000-S fluorescence microscope (Nikon, Tokyo, Japan). Images were captured using a digital camera (Hamamatsu Photonics, Hamamatsu, Japan) with a $60 \mathrm{X}$ oil immersion or $4 \mathrm{X}$ objective lens. Fluorescence intensity was analyzed using the ImageJ analysis system v1.45 (National Institutes of Health, Bethesda, MD, USA). In brief, the expression of TIAM1 and $\alpha$-SMA in BLM-treated mice was normalized to the expression in control mice. The number of FSP1(+) cells was quantified, and subsequently the expression of TIAM1 in FSP1(+) cells was analyzed based on the intensity of the merged color. 
WI-38 lung fibroblasts were purchased from the American Type Culture Collection (ATCC; Manassas, VA, USA). Cells were fixed with $3.7 \%$ formaldehyde for $10 \mathrm{~min}$ at $25^{\circ} \mathrm{C}$, treated with $0.25 \%$ Triton $\mathrm{X}-100$ for $10 \mathrm{~min}$ and subsequently blocked with $2.5 \%$ BSA (Santa Cruz Biotechnology, Inc.) at $25^{\circ} \mathrm{C}$ for $1 \mathrm{~h}$. Following blocking, cells were incubated at $25^{\circ} \mathrm{C}$ with rabbit anti-TIAM1 (1:200) and mouse anti- $\alpha$-SMA (1:400) antibodies for $1 \mathrm{~h}$. Cells were washed three times with PBS and subsequently incubated at $25^{\circ} \mathrm{C}$ with Alexa Fluor-tagged secondary antibodies (1:200, cat. no. R37117 and A-21202; Thermo Fisher Scientific, Inc.) at $25^{\circ} \mathrm{C}$ for $1 \mathrm{~h}$. Finally, the cells were mounted and examined using a Nikon Eclipse TE2000-S fluorescence microscope using a $60 \mathrm{X}$ oil immersion lens.

Cell culture and TGF- $\beta$ treatment. Primary mouse lung fibroblasts were isolated from $\mathrm{C} 57 \mathrm{BL} / 6 \mathrm{~J}$ mice with or without BLM treatment as previously described (17). Isolated mouse lung fibroblasts and human WI-38 fibroblasts were seeded and maintained in 6-well plates with Dulbecco's modified Eagle's medium (DMEM) containing 10\% FBS (Sigma-Aldrich; Merck KGaA). Following serum starvation for $24 \mathrm{~h}$, lung fibroblast cells $(\sim 80 \%$ confluent) were treated with TGF- $\beta$ (5 $\mathrm{ng} / \mathrm{ml})$ or PBS for 0 to $48 \mathrm{~h}$.

RNA isolation and reverse transcription-quantitative polymerase chain reaction $(R T-q P C R)$. Following treatment with TGF- $\beta$ (5 ng/ml, $12 \mathrm{~h}$ ), total RNA was extracted from WI-38 cells and purified using TRIzol (Invitrogen; Thermo Fisher Scientific, Inc., Waltham, MA, USA). cDNA was synthesized $\left(46^{\circ} \mathrm{C}, 20 \mathrm{~min}\right)$ by using a cDNA synthesis kit (iScript cDNA synthesis kit, cat. no. 1708890) from Bio-Rad Laboratories, Inc. A total of $1 \mu \mathrm{g}$ RNA was converted to cDNA, and this was used for qPCR. A SYBR Green qPCR kit and a CFX96 Touch qPCR system (both from Bio-Rad Laboratories, Inc.) were used. For real time qPCR, the amplification reactions were performed in triplicate, and the thermal cycling conditions were as follows: $10 \mathrm{sec}$ at $95^{\circ} \mathrm{C}$ followed by 40 cycles of $5 \mathrm{sec}$ at $95^{\circ} \mathrm{C}$ and $30 \mathrm{sec}$ at $60^{\circ} \mathrm{C}$. GAPDH was used as the reference gene to normalize the expression levels of TIAM1, FN and $\alpha$-SMA. The primers used were as follows: TIAM1 forward, 5'-GATCCACAGGAACTCCGAAGT-3' and reverse, 5'-GCTCCCGAAGTCTTCTAGGGT-3'; FN forward, 5'-TCTGTGCCTCCTATCTATGTGC-3' and reverse, 5'-GAG GGACCACGACAACTCTTC-3'; $\alpha$-SMA forward, 5'-AAA AGACAGCTACGTGGGTGA-3' and reverse, 5'-GCCATG TTCTATCGGGTACTTC-3'; GAPDH forward, 5'-TGTGGG CATCAATGGATTTGG-3' and reverse, 5'-ACACCATGT ATTCCGGGTCAAT-3'. Gene expression was analyzed using CFX Manager software version 3.1 (Bio-Rad Laboratories, Inc.). The $2^{-\triangle \Delta C q}$ method was used for quantification (17).

$N F-\kappa B$ inhibitor treatment. Cells (human lung fibroblast, WI-38) were pretreated with $10 \mu \mathrm{M}$ Bay 11-7082 and vehicle solution ( $0.05 \%$ dimethylsulfoxide), for $1 \mathrm{~h}$. The cells were subsequently challenged with TGF- $\beta$ ( $5 \mathrm{ng} / \mathrm{ml})$ for a subsequent $48 \mathrm{~h}$ prior to analysis using western blotting.

Transfection of WI-38 cells with siRNA. RNA smart pool targeting human TIAM1 (si-TIAM1) was from GE Dharmacon, Inc. (Lafayette, CO, USA), and control scramble (sc)-RNA was obtained from Santa Cruz Biotechnology, Inc. Fibroblasts were seeded into 6-well plates until they reached a confluence of 50-60\%. They were then transfected with $200 \mathrm{nmol} / \mathrm{l}$ si-TIAM1 or sc-RNA using siRNA transfection reagent (Huperfect Transfection reagent, cat. no. 301704; Qiagen AB, Sollentuna, Sweden). Briefly, the siRNAs were diluted in $900 \mu \mathrm{l}$ basal DMEM and incubated with the cells for at $37^{\circ} \mathrm{C}$ for $6 \mathrm{~h}$. DMEM with $10 \%$ FBS was refreshed every $24 \mathrm{~h}$. At $48 \mathrm{~h}$ following transfection, the cells were used for TGF- $\beta$ treatment and analyzed via fluorescence microscopy or western blot analysis.

Plasmid transfection of WI-38 cells. WI-38 cells grown to $-70 \%$ confluence were transiently transfected with $3 \mu \mathrm{g} / \mathrm{ml}$ of plasmids using plasmid transfection reagent (Effectene Transfection reagent, cat. no. 301425; Qiagen AB) in serum-free DMEM medium at $25^{\circ} \mathrm{C}$ for $15 \mathrm{~min}$, and further incubated in DMEM medium with $10 \%$ FBS for 48 h. Cells were subsequently used for TGF- $\beta$ treatment and protein expression analysis by western blot analysis.

Western blot analysis. Cells were disrupted by incubated with cell lysis buffer (cat. no. 9806; Cell Signaling Technology, Inc.) for $10 \mathrm{~min}$ on ice followed by centrifugation $(5,000 \mathrm{x} \mathrm{g}$, $15 \mathrm{~min}$ at $4^{\circ} \mathrm{C}$ ). Protein content in cell lysate was determined by using Micro BCA Protein Assay kit (cat. no. 23235; Thermo Fisher Scientific, Inc.). Briefly, 20-30 $\mu$ g proteins in cell lysates were separated by SDS-PAGE (10 or 4-20\%). Proteins were transferred onto nitrocellulose membranes $(100 \mathrm{~V}, 1 \mathrm{~h})$, blocked with blocking buffer (TBS solution containing $0.1 \%$ Tween-20 and 5\% BSA) at $25^{\circ} \mathrm{C}$ for $1 \mathrm{~h}$ and then incubated with primary antibodies (anti-TIAM1, 1:1,000; anti-FN, 1:2,000; anti-GAPDH, 1:2,000; anti- $\alpha$-SMA, 1:5,000) overnight at $4^{\circ} \mathrm{C}$. The membranes were subsequently incubated with secondary antibodies $(1: 2,000)$ at $25^{\circ} \mathrm{C}$ for $2 \mathrm{~h}$. Secondary antibodies used were horseradish peroxidase (HRP)-conjugated anti-mouse IgG (cat. no. 170-6515) and HRP-conjugated anti-rabbit IgG (cat. no. 170-6516) (both from Bio-Rad Laboratories, Inc.). Finally, proteins were visualized using an enhanced chemiluminescence kit (Bio-Rad Laboratories, Inc.) and analyzed using ImageQuant 5.2 software (Molecular Devices, LLC, Sunnyvale, CA, USA).

Statistical analysis. Data were analyzed using SPSS v16.0 statistical software (SPSS Inc., Chicago, IL, USA). Data are expressed as the mean \pm standard error of the mean from at least three independent experiments. Data were analyzed using a two-tailed Student t-test or two-way analysis of variance plus a multiple comparisons post-hoc test. $\mathrm{P}<0.05$ was considered to indicate a statistically significant difference.

\section{Results}

Expression of TIAM1 in mouse lung tissue. The expression of TIAM1 in lung tissues of mice with or without BLM-challenge (2 U/kg) was assessed on day 21. BLM treatment markedly induced lung injury and fibrosis in the wild-type mice, as indicated by increased cell infiltration into alveoli (Fig. 1A) and deposition of collagen (Fig. 1B) compared with control mice. Immunofluorescence (Fig. 1C and D) indicated that the 
A

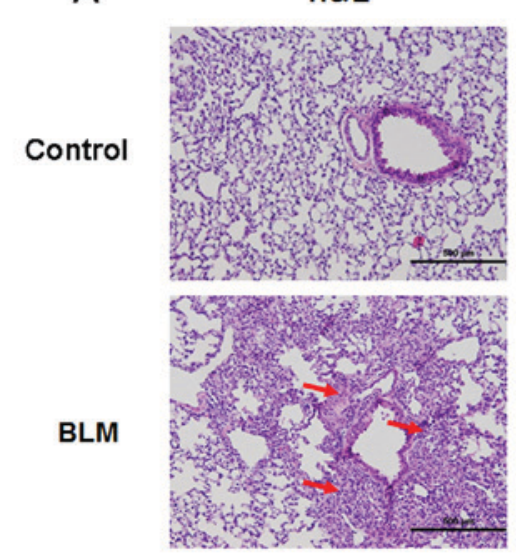

B

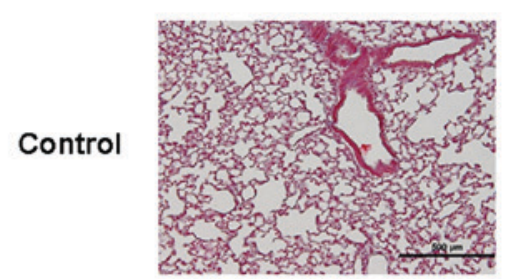

BLM

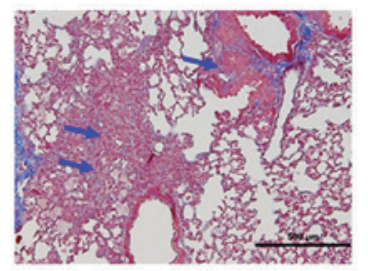

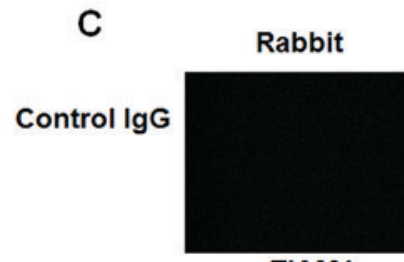

TIAM1
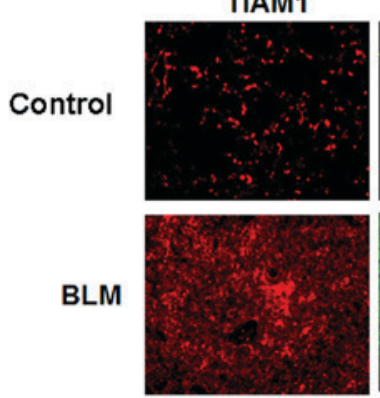

D
Mouse

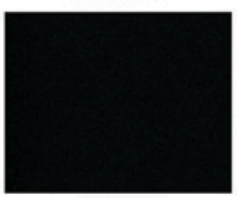

a-SMA
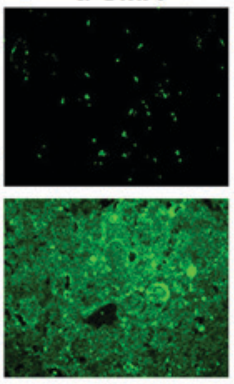

DAPI

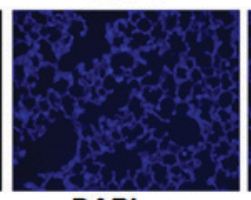

DAPI
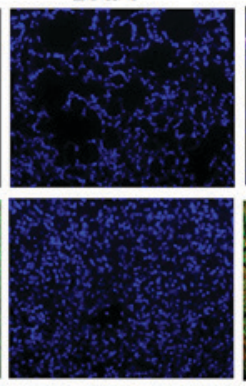

Merge

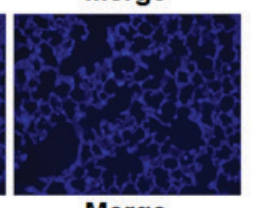

Merge
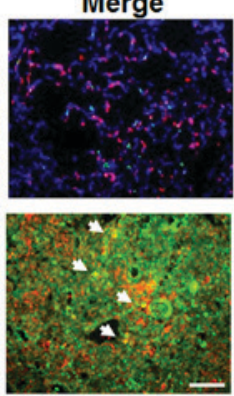

Control
BLM

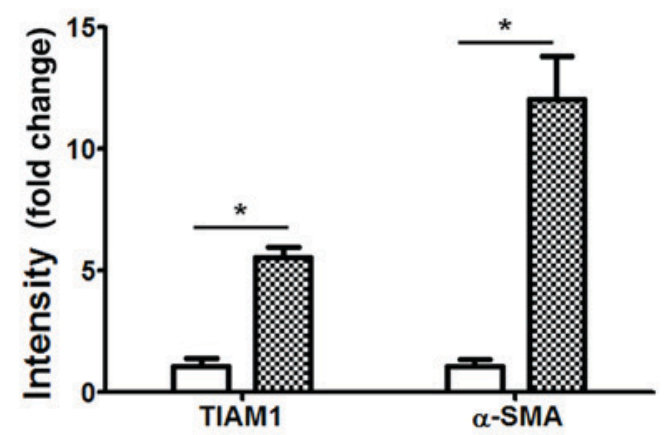

Figure 1. Expression of TIAM1 in lung tissues from control and BLM-treated mice. (A) H\&E staining (scale bar, $500 \mu \mathrm{m}$ ), (B) trichrome staining (scale bar, $500 \mu \mathrm{m}$ ) and (C) immunofluorescence staining of TIAM1, $\alpha$-SMA and DAPI (scale bar, $200 \mu \mathrm{m}$ ) in lung tissue from BLM-treated and control mice. Red arrows indicate lung injury, blue arrows indicate collagen deposition and white arrows indicate colocalized TIAM1 and $\alpha$-SMA overexpression in fibrotic foci. (C) The upper panel shows the representative immunofluorescence staining of negative control using mouse and rabbit control IgG. (D) Quantification of immunofluorescence staining. "P<0.05 as indicated. TIAM1, T-cell lymphoma invasion and metastasis 1; BLM, bleomycin; H\&E, hematoxylin and eosin; $\alpha$-SMA, $\alpha$-smooth muscle actin; IgG, immunoglobulin G.

expression of TIAM1 and $\alpha$-SMA was significantly increased in the lung fibrotic foci from BLM-challenged mice compared with control mice $(\mathrm{P}<0.05)$.

Expression of TIAM1 in mouse lung fibroblasts. To investigate the expression of TIAM1 in mouse lung fibroblasts, immunofluorescence staining for TIAM1 and FSP1 was performed in mouse lung tissues from control and BLM-treated mice. Staining indicated that the number FSP1(+) cells was significantly increased in the fibrotic foci from BLM-treated mice compared with control mice ( $\mathrm{P}<0.05$; Fig. $2 \mathrm{~A}$ and $\mathrm{B})$, and the expression of TIAM1 was significantly increased in the 
A

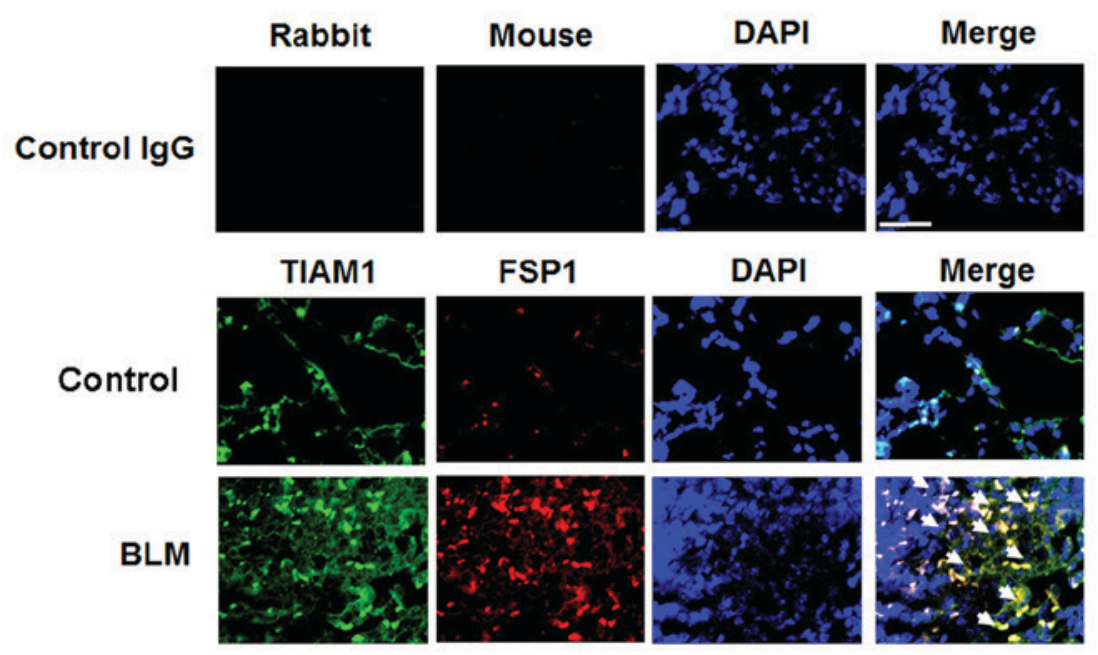

B

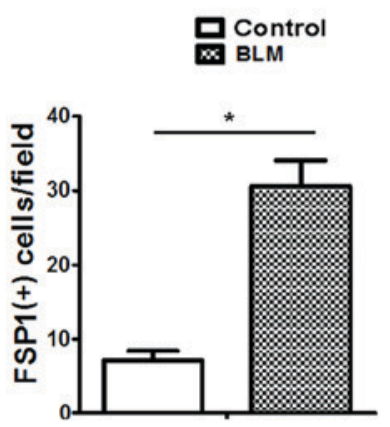

C

D

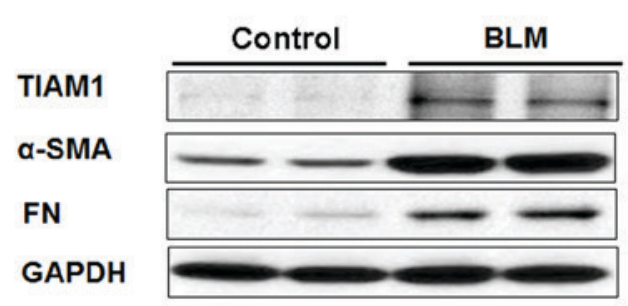

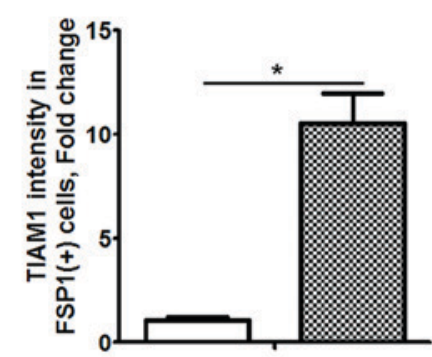

$\mathbf{E}$

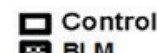

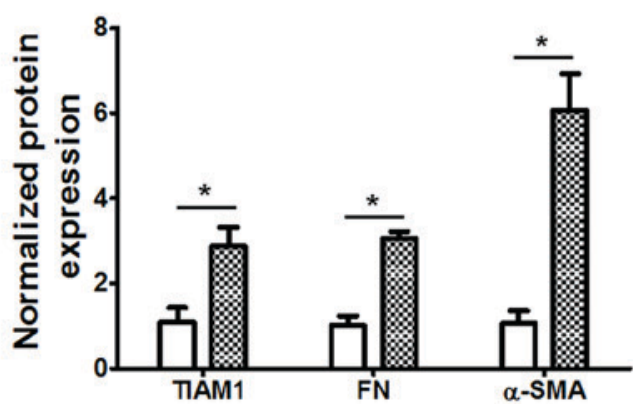

Figure 2. TIAM1 expression in lung fibroblasts from control and BLM-treated mice. (A) Representative immunofluorescence staining of TIAM1, FSP1 and DAPI in mouse lung tissue from control and BLM-treated mice. White arrows indicate colocalized overexpression of TIAM1 and FSP1 in fibrotic foci (scale bar, $50 \mu \mathrm{m}$ ). The upper panel is the representative immunofluorescence staining of negative control using mouse and rabbit control IgG. Quantification of the number of (B) FSP1(+) cells per field and (C) the expression of TIAM1 in FSP1(+) cells. (D) Western blot analysis and (E) quantified data for the expression of TIAM1, $\alpha$-SMA and FN in primary lung fibroblasts from control and BLM-treated mice. The protein expression was quantified and normalized to GAPDH. ${ }^{*} \mathrm{P}<0.05$ as indicated. TIAM1, T-cell lymphoma invasion and metastasis 1; BLM, bleomycin; FSP1, fibroblast specific protein 1; $\alpha$-SMA, $\alpha$-smooth muscle actin; FN, fibronectin.

FSP1(+) cells $(\mathrm{P}<0.05$; Fig. 2A and C). Western blot results also indicated that the expression levels of TIAM1, $\alpha$-SMA and FN were significantly higher in lung fibroblasts isolated from BLM-challenged mice compared with those from control mice ( $\mathrm{P}<0.05$; Fig. 2D and E). These data suggest that TIAM1 expression in lung fibroblasts may be associated with fibroblast differentiation in pulmonary fibrosis.
TIAM1 expression in TGF- $\beta$-treated human lung fibroblasts. Human fibroblasts were challenged with TGF- $\beta$ (5 ng/ml, $48 \mathrm{~h}$ ) and the results demonstrated that TGF- $\beta$ significantly increased the mRNA and protein levels of FN and $\alpha$-SMA $(\mathrm{P}<0.05$; Fig. 3A-C), indicating increased fibroblast differentiation compared with control cells. Similarly, cells challenged with TGF- $\beta$ had significantly increased mRNA 
A
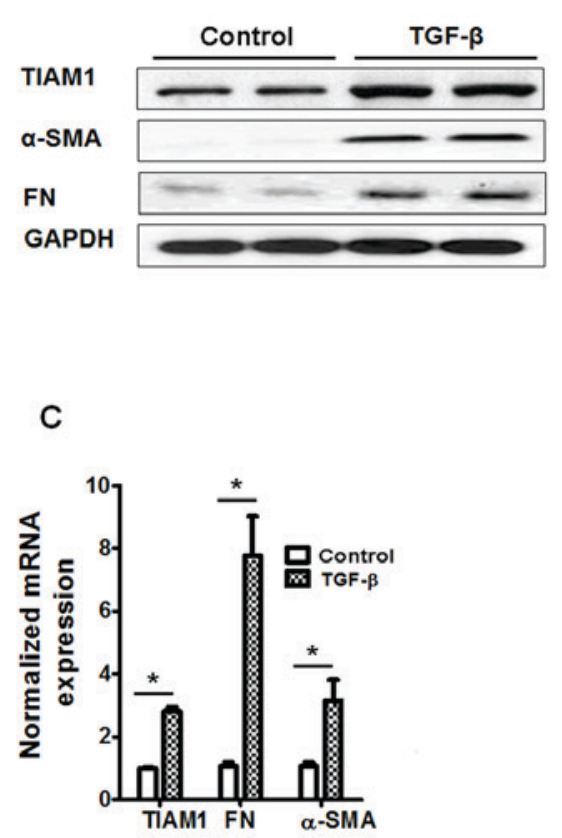

B 咱 Control

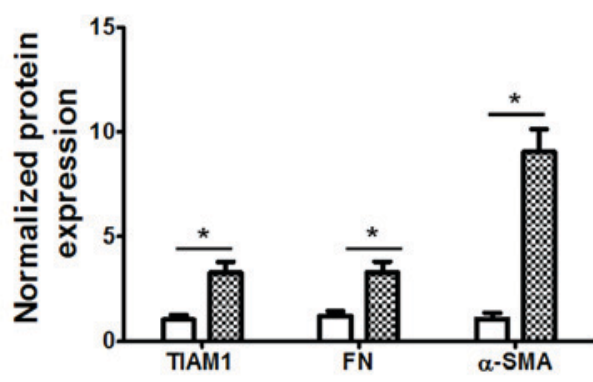

D
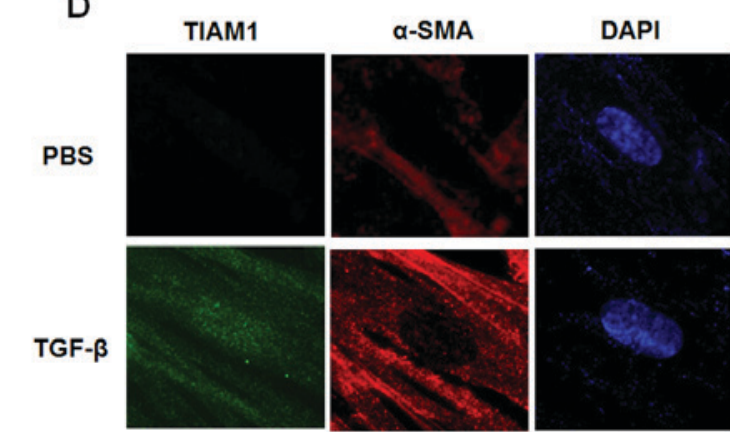

Merge
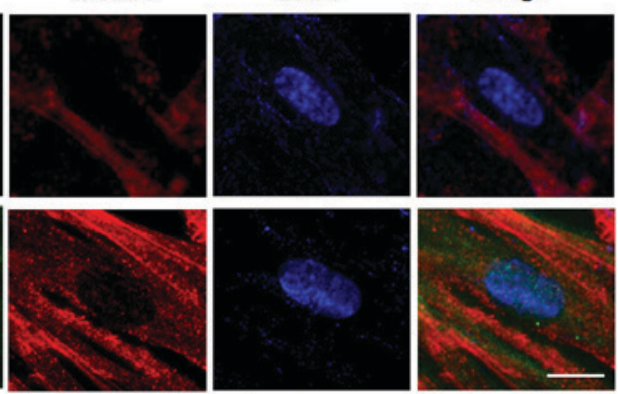

Figure 3. TGF- $\beta$ induces TIAM1 expression in human lung fibroblasts. WI-38 human lung fibroblasts were starved for 24 h and challenged with TGF- $\beta$ for 48 h. (A) Representative western blots and (B) quantification of the expression of TIAM1, FN and $\alpha$-SMA in human lung fibroblasts with or without TGF- $\beta$ challenge. (C) Reverse transcription-quantitative polymerase chain reaction analysis of gene expression and (D) immunofluorescence staining of $\alpha$-SMA and TIAM1 in WI-38 cells with TGF- $\beta$ challenge (scale bar, $10 \mu \mathrm{m}$ ). ${ }^{*} \mathrm{P}<0.05$ as indicated. TGF- $\beta$, transforming growth factor- $\beta$; TIAM1, T-cell lymphoma invasion and metastasis 1 ; FN, fibronectin; $\alpha$-SMA, $\alpha$-smooth muscle actin.

A

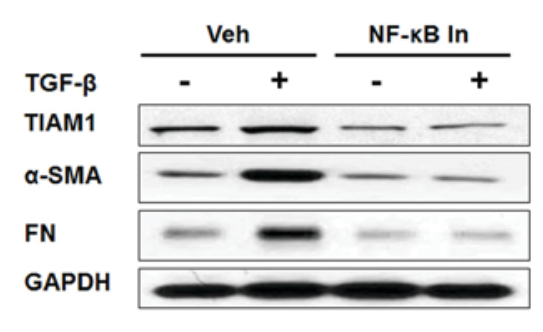

B

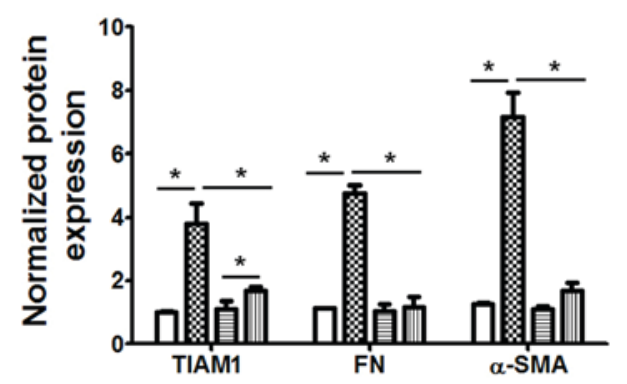

Figure 4. NF- $\mathrm{B}$ In attenuates TGF- $\beta$-induced fibroblast differentiation and TIAM1 expression in human lung fibroblasts. WI-38 cells were treated with Veh or NF- $\kappa$ B In prior to treatment with TGF- $\beta$. (A) Representative western blots and (B) quantification of TIAM1, FN and $\alpha-S M A$ expression. ${ }^{*} \mathrm{P}<0.05$ as indicated.

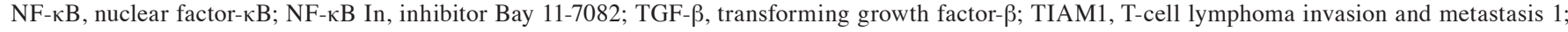
FN, fibronectin; $\alpha$-SMA, $\alpha$-smooth muscle actin; Veh, dimethyl sulfoxide.

and protein expression of TIAM1 compared with control cells $(\mathrm{P}<0.05$; Fig. 3A-C). Furthermore, immunofluorescence staining indicated that the TGF- $\beta$ challenge increased the expression of TIAM1 in human lung fibroblasts (Fig. 3D).

Inhibition of the $N F-\kappa B$ pathway attenuates TGF- $\beta$-induced fibroblast differentiation and TIAM1 expression in human lung fibroblasts. It has previously been indicated that inhibiting the $\mathrm{NF}-\kappa \mathrm{B}$ pathway via NF- $\kappa \mathrm{B}$ inhibitor treatment or knockdown of the NF- $\kappa \mathrm{B}$ p65 subunit blocks TGF- $\beta$-induced differentiation in human lung fibroblasts (17). In the present study, fibroblasts were treated with Bay 11-7082, then exposed to TGF- $\beta$ treatment and assessed using western blotting. Bay 11-7082 treatment was demonstrated to significantly attenuate TGF- $\beta$-induced fibroblast differentiation ( $\mathrm{P}<0.05$; Fig. 4) and markedly increase TIAM1 expression in human lung fibroblasts (Fig. 4A). These data suggest that TGF- $\beta$ induces TIAM1 expression in human lung fibroblasts via an NF- $\mathrm{B}$-dependent pathway and may also be associated with fibroblast differentiation.

TIAM1 inhibits TGF- $\beta$-induced human lung fibroblast differentiation. To assess the role of TIAM1 in TGF- $\beta$-induced 
A

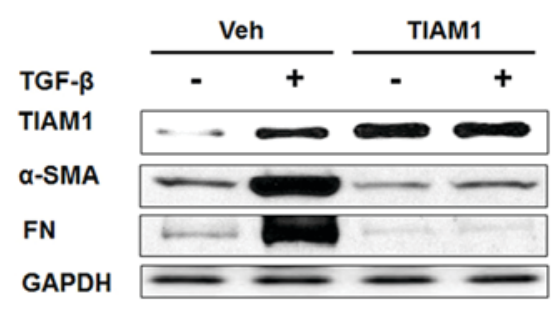

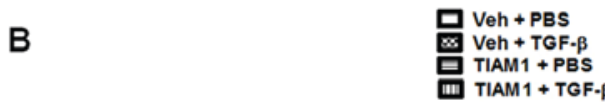

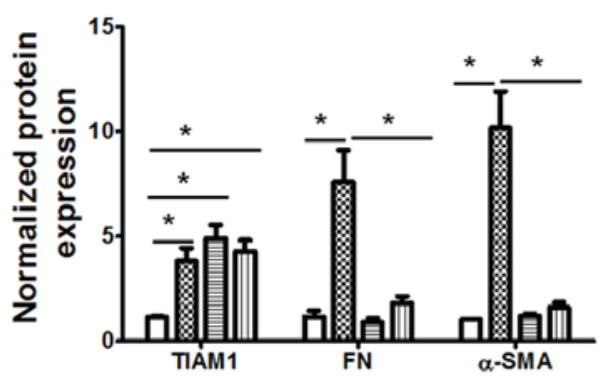

Figure 5. TIAM1 overexpression attenuates TGF- $\beta$-induced differentiation in human lung fibroblasts. WI-38 cells were transfected with Veh plasmid or TIAM1 plasmid and subsequently treated with TGF- $\beta$. (A) Representative western blots and (B) quantification of TIAM1, FN and $\alpha$-SMA expression. ${ }^{*} \mathrm{P}<0.05$ as indicated. TIAM1, T-cell lymphoma invasion and metastasis 1 ; TGF- $\beta$, transforming growth factor- $\beta$; FN, fibronectin; $\alpha$-SMA, $\alpha$-smooth muscle actin; Veh, vehicle.

A

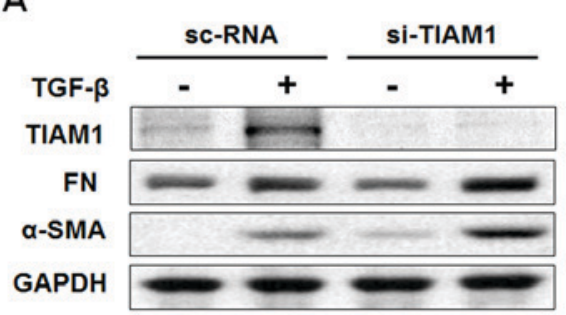

B

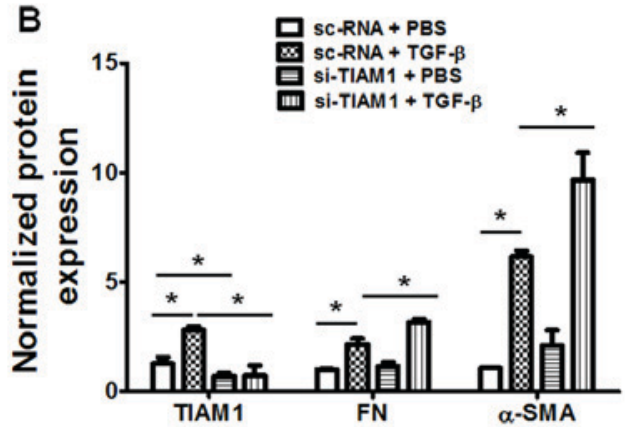

C

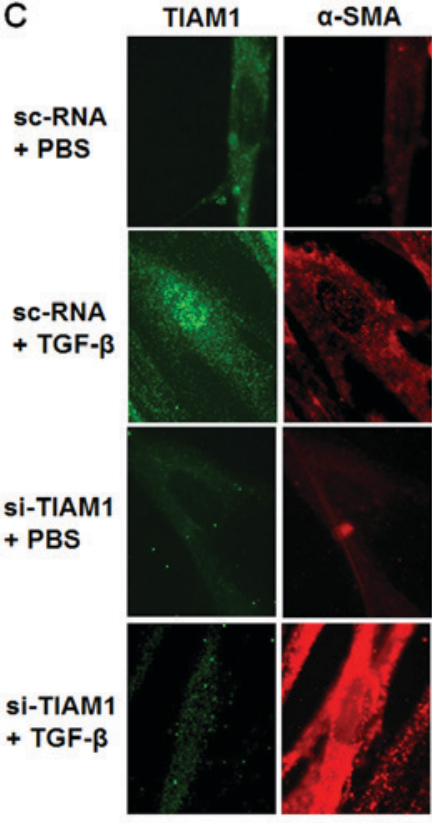

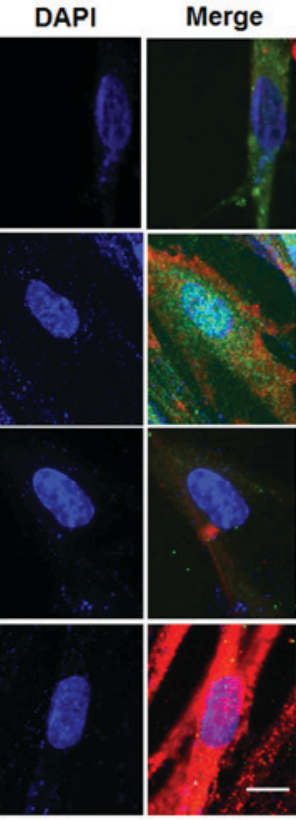

Figure 6. TIAM1 knockdown enhances TGF- $\beta$-induced differentiation in human lung fibroblasts. WI-38 cells were transfected with sc-RNA or si-TIAM1 and subsequently treated with TGF- $\beta$. (A) Representative western blots and (B) quantification of TIAM1, FN and $\alpha$-SMA expression and (C) immunofluorescence staining of $\alpha$-SMA and TIAM1 (scale bar, $10 \mu \mathrm{m}$ ). ${ }^{*} \mathrm{P}<0.05$ as indicated. TIAM1, T-cell lymphoma invasion and metastasis 1 ; TGF- $\beta$, transforming growth factor- $\beta$; sc-RNA, scramble RNA; si-TIAM1, TIAM1 short interfering RNA; FN, fibronectin; $\alpha$-SMA, $\alpha$-smooth muscle actin.

fibroblast differentiation, human lung fibroblasts were transfected with plasmids coding human TIAM1 protein. Transfection with the TIAM1 plasmid markedly increased the expression of TIAM1 protein in human lung fibroblasts (Fig. 5A). Furthermore, TIAM1 overexpression was observed to significantly inhibit the TGF- $\beta$-induced overexpression of FN and $\alpha$-SMA, indicating that lung fibroblast differentiation was significantly reduced compared with that of control cells $(\mathrm{P}<0.05$; Fig. 5). The effect of TIAM1 knockdown was also assessed via siRNA transfection. It was demonstrated that TIAM1 knockdown significantly increased the TGF- $\beta$-induced expression of FN and $\alpha$-SMA in human lung fibroblasts $(\mathrm{P}<0.05$; Fig. 6). Together, these data suggest that TIAM1 inhibits TGF- $\beta$-induced human lung fibroblast differentiation.

\section{Discussion}

TIAM1 is a guanine nucleotide exchange factor, which is crucially involved in tumor cell invasion and migration $(20,21)$. Clinical studies have suggested that TIAM1 overexpression predicates poor overall survival in patients with primary gallbladder carcinoma (22), prostate cancer (23) and hepatocellular carcinoma (24). In vitro studies in cancer cell lines have indicated that the TIAM1 gene serves important roles in the proliferation, invasion and metastasis of giant-cell lung carcinoma (5), human breast cancer (20), hepatocellular carcinoma (25) and colorectal cancer (26). However, there is limited information available on the effects of TIAM1 on pulmonary fibrosis. The results of the present study demonstrate that TIAM1 expression is associated with pulmonary 
fibrosis in a BLM-induced mouse model of pulmonary fibrosis. Immunostaining and western blotting data indicate that the expression of TIAM1 is significantly higher in fibroblasts from fibrotic lung tissue compared with those from controls. These data suggest that TIAM1 serves an important role in pulmonary fibrosis.

Fibroblasts are activated following tissue injury. In physiological conditions, activated fibroblasts produce ECM proteins followed by apoptosis; however, under pathological conditions, including in IPF, the activated fibroblasts resist apoptosis and exhibit high proliferation and differentiation $(13,27,28)$. Activated fibroblasts induce the expression and secretion of TGF- $\beta$ and fibroblast growth factor $(13,29,30)$, which stimulate growth and proliferation in various cells, including fibroblasts and breast cancer cells (31). In cancer tissues, carcinoma-associated fibroblasts highly express $\alpha$-SMA, fibroblast activation protein and fibroblast surface protein, which are protein markers of differentiated fibroblasts (32). Furthermore, activation of carcinoma-associated fibroblasts has been demonstrated to inhibit apoptosis in hepatocellular carcinoma cells (32). These observations suggest that fibroblast differentiation and activation are critical to the resistance of cancer cells to apoptosis.

Previous studies have indicated that the molecular mechanisms of IPF may be associated with lung cancer $(33,34)$. Elderly male cigarette smokers with IPF were demonstrated to be at a higher risk of developing lung cancer, and tumors were more commonly observed in the lower lobes and peripheral regions of lungs (35). The results of the present study suggest that the oncogene TIAM1 also serves critical roles in pulmonary fibrosis, specifically in lung fibroblasts. They also indicate that the expression of TIAM1 is associated with differentiation of lung fibroblasts, and that TGF- $\beta$, which is a key factor of pulmonary fibrosis, directly stimulates fibroblast differentiation and TIAM1 expression via an NF- $\mathrm{B}$-dependent pathway. A previous study of lung fibroblasts indicated that differentiation may be associated with cell invasion and lung fibrogenesis (36), which suggests that TIAM1 may be essential for fibrotic phenotype changes in differentiated lung fibroblasts. TIAM1 knockdown in fibroblasts has previously been reported to enhance the invasion of lung cancer cells in vitro (5). The results of the present study revealed that TIAM1 overexpression blocks the TGF- $\beta$-induced differentiation of fibroblasts, which may decrease fibrosis in vivo. Furthermore, knocking down the expression of endogenous TIAM1 further augments fibroblast differentiation. These data suggest that TIAM1 serves different roles in fibroblast differentiation and cancer cell invasion. However, further investigation is required to elucidate the role of TIAM1 in fibroblast migration and invasion.

The present study indicates that the expression of TIAM1 may be dependent on fibroblast differentiation. Differentiated fibroblasts express higher level of ECM proteins, including collagen and FN, compared with undifferentiated fibroblasts $(12,13)$. In pancreatic $\beta$-cells, ECM proteins stimulate cell proliferation, and induce $\mathrm{NF}-\kappa \mathrm{B}$ nuclear translocation and the expression of NF- $\kappa \mathrm{B}$ inhibitor (37). Furthermore, blocking the NF- $\kappa \mathrm{B}$ pathway completely blocks ECM-induced cell proliferation (37) and the ECM-induced $N F-\kappa B$ pathway is essential for maintaining glucose-induced expression and the secretion of insulin in pancreatic $\beta$-cells (38). However, the effect of ECM in the NF- $\mathrm{NB}$ pathway in fibroblasts remains to be elucidated. The results of the present study demonstrate that inhibiting the NF- $\kappa \mathrm{B}$ pathway blocks the TGF- $\beta$-induced expression of FN and TIAM1, and indicated that TIAM1 expression depends on $\mathrm{NF}-\kappa \mathrm{B}$ activation. During lung fibrogenesis, differentiated fibroblasts display higher expression level and nuclear translocation of the NF- $\mathrm{KB}$ p65 subunit (39), which suggests that differentiated fibroblasts may induce activation of the $\mathrm{NF}-\kappa \mathrm{B}$ pathway. However, the underlying molecular mechanisms require further study.

In conclusion, the results of the present study suggest that TIAM1, an oncogene, is also associated with pulmonary fibrosis. The expression of TIAM1 is upregulated in fibroblasts during differentiation, whereas overexpression of TIAM1 attenuates TGF- $\beta$-induced differentiation in human lung fibroblasts. The data in the present study indicate that TIAM1 serves critical roles in BLM-induced lung fibrosis in mice, and inhibits the differentiation of pulmonary fibroblasts. These data suggest that TIAM1 is a potential therapeutic target in pulmonary fibrosis.

\section{References}

1. Bourguignon LY, Zhu H, Shao L and Chen YW: Ankyrin-Tiam1 interaction promotes Racl signaling and metastatic breast tumor cell invasion and migration. J Cell Biol 150: 177-191, 2000.

2. Ceccarelli DF, Blasutig IM, Goudreault M, Li Z, Ruston J, Pawson T and Sicheri F: Non-canonical interaction of phosphoinositides with pleckstrin homology domains of Tiam1 and ArhGAP9. J Biol Chem 282: 13864-13874, 2007.

3. LeeuwenFN, Kain HE, Kammen RA, Michiels F, Kranenburg OW and Collard JG: The guanine nucleotide exchange factor Tiam1 affects neuronal morphology; opposing roles for the small GTPases Rac and Rho. J Cell Biol 139: 797-807, 1997.

4. Paliwal S, Ho N, Parker D and Grossman SR: CtBP2 promotes human cancer cell migration by transcriptional activation of tiam1. Genes Cancer 3: 481-490, 2012.

5. Wang HM and Wang J: Expression of Tiam1 in lung cancer and its clinical significance. Asian Pac J Cancer Prev 13: 613-615, 2012.

6. Vaughan L, Tan CT, Chapman A, Nonaka D, Mack NA, Smith D, Booton R, Hurlstone AF and Malliri A: HUWE1 ubiquitylates and degrades the RAC activator TIAM1 promoting cell-cell adhesion disassembly, migration, and invasion. Cell Rep 10: 88-102, 2015.

7. Minard ME, Herynk MH, Collard JG and Gallick GE: The guanine nucleotide exchange factor Tiam 1 increases colon carcinoma growth at metastatic sites in an orthotopic nude mouse model. Oncogene 24: 2568-2573, 2005.

8. Mertens AE, Pegtel DM and Collard JG: Tiam1 takes PARt in cell polarity. Trends Cell Biol 16: 308-316, 2006.

9. Desai LP, Chapman KE and Waters CM: Mechanical stretch decreases migration of alveolar epithelial cells through mechanisms involving Rac1 and Tiam1. Am J Physiol Lung Cell Mol Physiol 295: L958-L965, 2008.

10. The Lancet Respiratory Medicine: The changing landscape of idiopathic pulmonary fibrosis. Lancet Respir Med 2: 507, 2014.

11. Wynn TA: Cellular and molecular mechanisms of fibrosis. J Pathol 214: 199-210, 2008.

12. Huang LS and Natarajan V: Sphingolipids in pulmonary fibrosis. Adv Biol Regul 57: 55-63, 2015.

13. Wynn TA: Integrating mechanisms of pulmonary fibrosis. J Exp Med 208: 1339-1350, 2011.

14. Leask A and Abraham DJ: TGF-beta signaling and the fibrotic response. FASEB J 18: 816-827, 2004.

15. Sime PJ, Xing Z, Graham FL, Csaky KG and Gauldie J: Adenovector-mediated gene transfer of active transforming growth factor-beta1 induces prolonged severe fibrosis in rat lung. J Clin Invest 100: 768-776, 1997.

16. Xu P, Liu J and Derynck R: Post-translational regulation of TGF- $\beta$ receptor and Smad signaling. FEBS Lett 586: 1871-1884, 2012. 
17. Livak KJ and Schmittgen TD: Analysis of relative gene expression data using real-time quantitative PCR and the 2(-Delta Delta C(T)) method. Methods 25: 402-408, 2001.

18. Song N, Liu J, Shaheen S, Du L, Proctor M, Roman J and Yu J: Vagotomy attenuates bleomycin-induced pulmonary fibrosis in mice. Sci Rep 5: 13419, 2015.

19. Choi SH, Kim M, Lee HJ, Kim EH, Kim CH and Lee YJ: Effects of NOX1 on fibroblastic changes of endothelial cells in radiation-induced pulmonary fibrosis. Mol Med Rep 13: 4135-4142, 2016.

20. Minard ME, Kim LS, Price JE and Gallick GE: The role of the guanine nucleotide exchange factor Tiam1 in cellular migration, invasion, adhesion and tumor progression. Breast Cancer Res Treat 84: 21-32, 2004

21. Liu S, Li Y, Qi W, Zhao Y, Huang A, Sheng W, Lei B, Lin P, Zhu H, Li W and Shen H: Expression of Tiam1 predicts lymph node metastasis and poor survival of lung adenocarcinoma patients. Diagn Pathol 9: 69, 2014.

22. Du X, Wang S, Lu J, Wang Q, Song N, Yang T, Dong R, Zang L, Yang Y, Wu T and Wang C: Clinical value of Tiam1-Racl signaling in primary gallbladder carcinoma. Med Oncol 29: 1873-1878, 2012

23. Engers R, Mueller M, Walter A, Collard JG, Willers R and Gabbert HE: Prognostic relevance of Tiam1 protein expression in prostate carcinomas. Br J Cancer 95: 1081-1086, 2006.

24. Huang J, Ye X, Guan J, Chen B, Li Q, Zheng X, Liu L, Wang S, Ding Y, Ding Y and Chen L: Tiam1 is associated with hepatocellular carcinoma metastasis. Int J Cancer 132: 90-100, 2013.

25. Ding Y, Chen B, Wang S, Zhao L, Chen J, Ding Y, Chen L and Luo R: Overexpression of Tiam1 in hepatocellular carcinomas predicts poor prognosis of HCC patients. Int J Cancer 124 653-658, 2009

26. Liu L, Wu DH and Ding YQ: Tiam1 gene expression and its significance in colorectal carcinoma. World J Gastroenterol 11: 705-707, 2005.

27. Nho RS and Polunovsky V: Translational control of the fibroblast-extracellular matrix association: An application to pulmonary fibrosis. Translation (Austin) 1: e23934, 2013.

28. Darby IA, Laverdet B, Bonté F and Desmoulieré A: Fibroblasts and myofibroblasts in wound healing. Clin Cosmet Investig Dermatol 7: 301-311, 2014.
29. Gohda E, Matsunaga T, Kataoka H, Takebe T and Yamamoto I: Induction of hepatocyte growth factor in human skin fibroblasts by epidermal growth factor, platelet-derived growth factor and fibroblast growth factor. Cytokine 6: 633-640, 1994

30. Strutz F, Zeisberg M, Renziehausen A, Raschke B, Becker V, van Kooten $C$ and Müller G: TGF-beta 1 induces proliferation in human renal fibroblasts via induction of basic fibroblast growth factor (FGF-2). Kidney Int 59: 579-592, 2001.

31. Andre F and Cortés J: Rationale for targeting fibroblast growth factor receptor signaling in breast cancer. Breast Cancer Res Treat 150: 1-8, 2015.

32. Song T, Dou C, Jia Y, Tu K and Zheng X: TIMP-1 activated carcinoma-associated fibroblasts inhibit tumor apoptosis by activating SDF1/CXCR4 signaling in hepatocellular carcinoma. Oncotarget 6: 12061-12079, 2015.

33. Mizuno K, Mataki H, Seki N, Kumamoto T, Kamikawaji K and Inoue H: MicroRNAs in non-small cell lung cancer and idiopathic pulmonary fibrosis. J Hum Genet 62: 57-65, 2017.

34. Stella GM, Inghilleri S, Pignochino Y, Zorzetto M, Oggionni T, Morbini P and Luisetti M: Activation of oncogenic pathways in idiopathic pulmonary fibrosis. Transl Oncol 7: 650-655, 2014.

35. Archontogeorgis K, Steiropoulos P, Tzouvelekis A, Nena E and Bouros D: Lung cancer and interstitial lung diseases: A systematic review. Pulm Med 2012: 315918, 2012.

36. Li Y, Jiang D, Liang J, Meltzer EB, Gray A, Miura R, Wogensen L, Yamaguchi Y and Noble PW: Severe lung fibrosis requires an invasive fibroblast phenotype regulated by hyaluronan and CD44. J Exp Med 208: 1459-1471, 2011.

37. Parnaud G, Hammar E, Ribaux P, Donath MY, Berney T and Halban PA: Signaling pathways implicated in the stimulation of beta-cell proliferation by extracellular matrix. Mol Endocrinol 23: 1264-1271, 2009.

38. Hammar EB, Irminger JC, Rickenbach K, Parnaud G, Ribaux P, Bosco D, Rouiller DG and Halban PA: Activation of NF-kappaB by extracellular matrix is involved in spreading and glucose-stimulated insulin secretion of pancreatic beta cells. J Biol Chem 280: 30630-30637, 2005.

39. Sun X, Chen E, Dong R, Chen W and Hu Y: Nuclear factor (NF)- $\mathrm{kB}$ p65 regulates differentiation of human and mouse lung fibroblasts mediated by TGF- $\beta$. Life Sci 122: 8-14, 2015. 